\title{
BMJ Open Reducing paediatric overweight and obesity through motivational interviewing: study protocol for a randomised controlled trial in the AAP PROS research network
}

\author{
Margaret E Wright (10 , , ${ }^{1,2}$ Emerson Delacroix, ${ }^{3}$ Kendrin R Sonneville, ${ }^{3}$ \\ Shannon Considine, ${ }^{3}$ Tim Proctor, ${ }^{4}$ Jennifer Steffes, ${ }^{1,2}$ Donna Harris, ${ }^{1,2}$ \\ Laura P Shone, ${ }^{1,2}$ Heide Woo, ${ }^{5}$ Roger Vaughan, ${ }^{6}$ Robert W Grundmeier, ${ }^{7}$ \\ Alexander G Fiks, ${ }^{2,7}$ Melissa S Stockwell, ${ }^{2,8}$ Ken Resnicow ${ }^{3}$
}

To cite: Wright ME, Delacroix E, Sonneville KR, et al. Reducing paediatric overweight and obesity through motivational interviewing: study protocol for a randomised controlled trial in the AAP PROS research network. BMJ Open 2020;10:e035720. doi:10.1136/ bmjopen-2019-035720

- Prepublication history and additional material for this paper are available online. To view these files, please visit the journal online (http://dx.doi org/10.1136/bmjopen-2019035720).

Received 12 November 2019 Revised 08 May 2020 Accepted 11 May 2020
Check for updates

(C) Author(s) (or their employer(s)) 2020. Re-use permitted under CC BY-NC. No commercial re-use. See rights and permissions. Published by BMJ.

For numbered affiliations see end of article.

Correspondence to Dr Margaret E Wright; mewright@uic.edu

\section{ABSTRACT}

Introduction Primary care remains an underused venue for prevention and management of paediatric overweight and obesity. A prior trial demonstrated a significant impact of paediatrician/nurse practitioner (Ped/NP)-and registered dietitian (RD)-delivered motivational interviewing (MI) on child body mass index (BMI). The study described here will test the effectiveness of an enhanced version of this primary care-based Ml counselling intervention on child BMI.

Methods and analysis This cluster randomised effectiveness trial includes 24 Ped/NPs from 18 paediatric primary care practices that belong to the American Academy of Pediatrics (AAP) national Pediatric Research in Office Settings (PROS) practice-based research network. To date, practices have been randomised (nine to intervention and nine to usual care). Intervention Ped/ NPs have been trained in MI, behavioural therapy, billing/ coding for weight management and study procedures. Usual care Ped/NPs received training in billing/coding and study procedures only. Children 3-11 years old with BMI $\geq$ the 85th percentile were identified via electronic health records (EHRs). Parents from intervention practices have been recruited and enrolled. Over about 2 years, these parents are offered approximately $10 \mathrm{Ml}$-based counselling sessions (about four in person sessions with their child's $\mathrm{Ped} / \mathrm{NP}$ and up to six telephonic sessions with a trained $\mathrm{RD}$ ). The primary outcome is change in child BMI (defined as per cent from median BMI for age and sex) over the study period. The primary comparison is between eligible children in intervention practices whose parents enrol in the study and all eligible children in usual care practices. Data sources will include EHRs, billing records, surveys and counselling call notes.

Ethics and dissemination Institutional Review Board approval was obtained from the AAP. All Ped/NPs provided written informed consent, and intervention group parents provided consent and Health Insurance Portability and Accountability Act (HIPAA) authorisation. Findings will be disseminated through peer-reviewed publications, conference presentations and appropriate AAP channels.
Strengths and limitations of this study

- A strength of the trial is that the intervention is being tested under real-world conditions among a sample of practices and families throughout the USA.

- Another strength is that we will examine intervention effectiveness among the population of all eligible children, in addition to the traditional evaluation among enrollees.

- A strength is that the primary outcome will be ascertained using data extracted from electronic health records (EHRs) of eligible children in participating practices, rather than relying on parent report.

- A limitation is that only parents of children 3-11 years old are included, which may limit generalisability to other age groups.

- A limitation is that the trial was restricted to practices that used one specific EHR vendor, which may limit generalisability.

Trial registration number NCT03177148; Pre-results.

\section{INTRODUCTION}

More than one-third of all children in the USA have overweight or obesity, with substantial disparities by race and ethnicity, income, education and geographical location. ${ }^{1-3}$ Most children with obesity remain so as adolescents and adults, ${ }^{4}$ facing a variety of health complications including diabetes, liver disease, asthma, heart disease and cancer. ${ }^{5}$ Childhood obesity is also linked to lower health-related quality of life, behaviour problems and psychosocial dysfunction. ${ }^{6} 7$ Rates of overweight and obesity among all children remain 2-3 times higher than 30 years ago. ${ }^{8}$

Paediatric healthcare professionals can play a crucial role in treating childhood 
obesity. Paediatricians provide the majority of primary medical care to children in the USA, advise families on nutrition, routinely monitor growth and have frequent contact with patients and their families. ${ }^{9}{ }^{10}$ Paediatric primary care offers an important opportunity to address weight concerns at an earlier age, and to monitor weight status over time. Expert Committee Recommendations endorsed by the American Academy of Pediatrics (AAP) highlight a four-stage treatment approach, of which the first two can occur routinely in primary care: stage 1 ('prevention-plus') focuses on healthy lifestyle changes and stage 2 entails structured weight management. ${ }^{11} 12$

Recent reviews show mixed results across paediatric behavior-based weight loss intervention trials in primary care. ${ }^{13}$ One promising approach is motivational interviewing (MI). MI is a patient-centred counselling style that originated for use with adults to treat substance use ${ }^{14}$ and has since been used by paediatric and adult healthcare professionals to address a wide range of conditions and behaviours including nutrition, physical activity and other behaviours. ${ }^{15} 16$ Although MI has shown promise in paediatric settings, few paediatricians receive formal training in MI (though many have expressed strong interest in it), ${ }^{17}$ and many report feeling that their counselling on obesity management is not effective. ${ }^{17} 18$

The study described here is called Population Effects of Motivational Interviewing on Pediatric Obesity in Primary Care. This randomised controlled trial is funded by the National Heart, Lung and Blood Institute of the National Institutes of Health, and led by the University of Michigan. The AAP, Children's Hospital of Philadelphia (CHOP) and Physician's Computer Company (PCG) are collaborators on the study.

The prior efficacy trial, which forms the basis for the current study, was called Brief Motivational Interviewing to Reduce Body Mass Index $\left(\mathrm{BMI}^{2}\right) .{ }^{19} \mathrm{BMI}^{2}$ showed statistically and clinically significant reductions in BMI percentile in children 2-8 years old with overweight or obesity whose parents received MI counselling during up to 10 sessions with their child's paediatrician or nurse practitioner (Ped/NP) and a registered dietitian (RD) compared with usual care over 2 years. ${ }^{20}$ Specifically, the observed BMI percentile reduction in the prior study falls within the range that other studies have shown to have clinically meaningful impacts on biomarkers of insulin sensitivity and cardiometabolic health. ${ }^{21} 22$

The current study is testing the effectiveness of an enhanced version of the $\mathrm{BMI}^{2}$ intervention, and is therefore referred to as $\mathrm{BMI}^{2+}$. As a pragmatic effectiveness trial, the number of MI counselling sessions (up to 10), duration of follow-up (approximately 2 years), and comparator (usual care) remain the same in $\mathrm{BMI}^{2+}$. Enhancements in the $\mathrm{BMI}^{2+}$ study include: (1) remote telephone counselling for paediatric weight management from centrally employed, trained and supervised RDs; (2) use of web based, interactive portals for RDs, Ped/ NPs and enrolled parents; (3) text message reminders and tailored behavioural reinforcement messages for

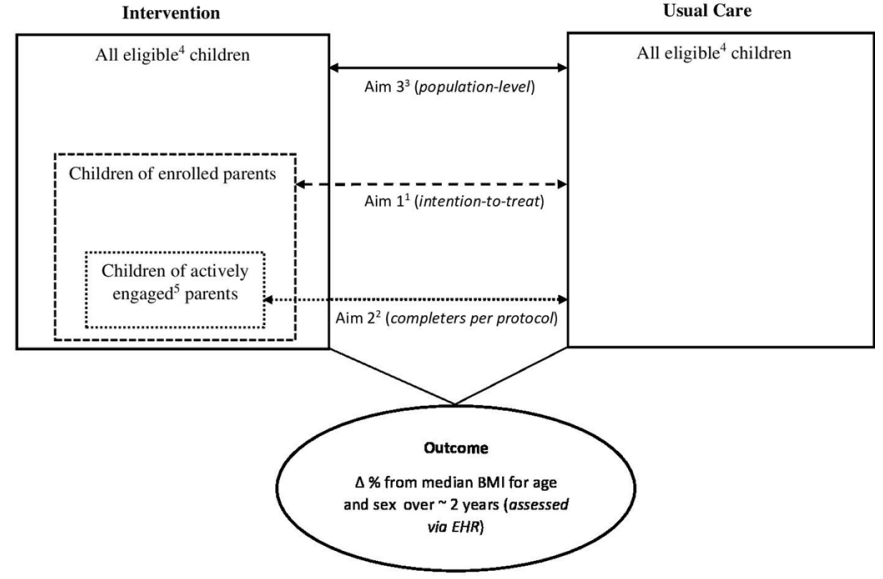

Figure $1 \mathrm{BMI}^{2+}$ study aims and comparisons. ${ }^{1}$ Compare outcome between children of enrolled parents (intervention) and all eligible children (usual care). ${ }^{2}$ Compare outcome between children of actively engaged parents (intervention) and all eligible children (usual care). ${ }^{3}$ Compare outcome between all eligible children in both intervention and usual care. ${ }^{4}$ Eligibility based on age (3-11 years), BMI (>85th percentile), health supervision history (visit within 2 years of baseline with a participating Ped/NP) and medical history (no chronic, limiting, severe medical disorder or use of medications known to affect grown and mood/behaviour). ${ }^{5}$ Defined as receipt of at least $50 \%$ of the total Ml counselling sessions from Ped/NPs and RDs. BMI, body mass index; EHR, electronic health record; MI, motivational interviewing; $\mathrm{Ped} / \mathrm{NP}$, paediatrician/nurse practitioner; RD, registered dietitians.

enrolled parents; (4) customisation and utilisation of electronic health records (EHRs) to identify eligible families and track child BMI over time and (5) analysis of intervention effectiveness among the population of all eligible children, in addition to the traditional evaluation among enrollees.

\section{Aims}

The primary and secondary study aims are shown in figure 1 . The primary aim is to test the effectiveness of the $\mathrm{BMI}^{2+}$ intervention versus usual care on change in child BMI, defined as the per cent from median BMI for age and sex over approximately 2 years. Eligible patients of participating Ped/NPs whose parents enrolled in the study (intervention) will be compared with eligible children in usual care practices (intention-to-treat analysis). The secondary aims are to compare changes in BMI in (1) the subgroup of eligible patients of participating Ped/NPs whose parents actively participated in the intervention (defined as receipt of at least $50 \%$ of the total MI counselling sessions from Ped/NPs and RDs) versus all eligible children in usual care practices (analysis of completers per protocol) and (2) all eligible children of participating Ped/NPs in intervention practices, even if the child's parent did not enrol in the study, versus all eligible children in usual care practices (population-level analysis). 


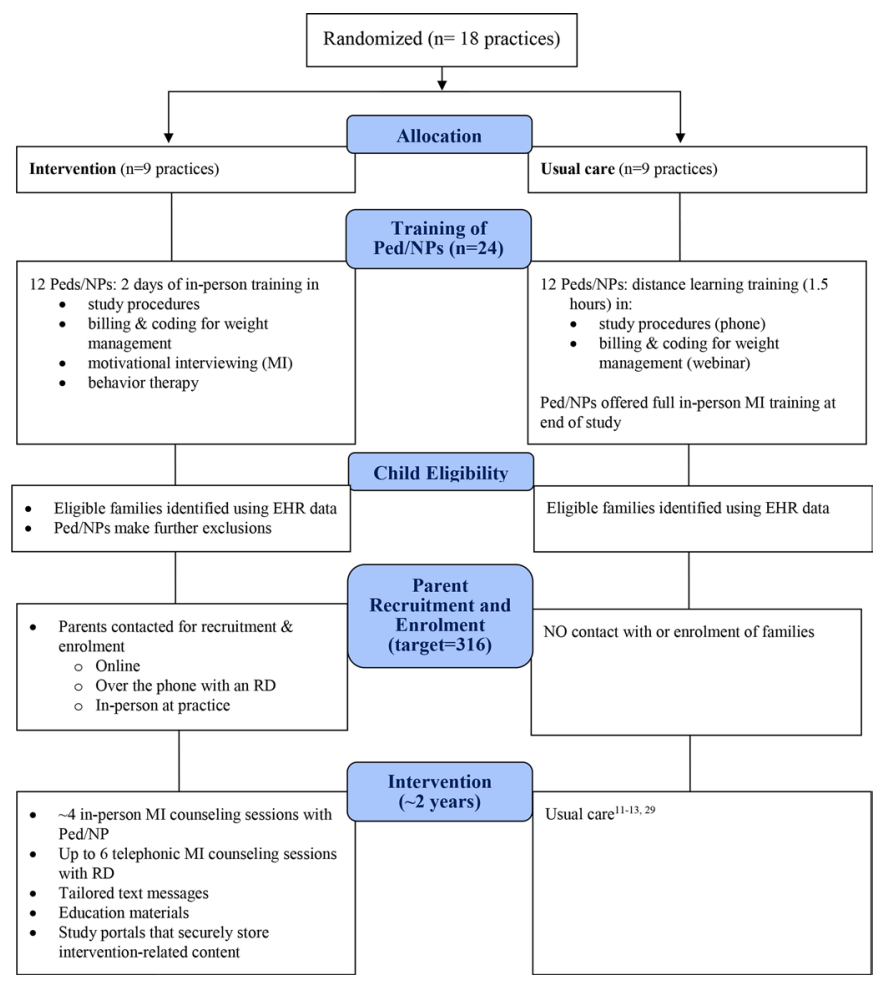

Figure 2 Overview of the $\mathrm{BMI}^{2+}$ study. BMI, body mass index; EHR, electronic health record; Ped/NP, paediatrician/ nurse practitioner; $\mathrm{RD}$, registered dietitian.

\section{METHODS AND ANALYSIS}

\section{Trial design}

This study is a two-group cluster randomised trial with practices serving as the unit of randomisation and analysis (figure 2). Eighteen practices were randomised (nine to intervention and nine to usual care) in 2017, in-person training of 12 intervention Ped/NPs took place October through December 2017, and telephone/webinar training of 12 usual care Ped/NPs was completed shortly thereafter. Parents were recruited and enrolled in intervention group practices between November 2017 and April 2019, and intervention delivery and data collection will be complete by 2021 . Table 1 presents the schedule of enrolment, training and intervention delivery. Although the primary outcome of interest is change in child BMI, parents are the primary recipient of the MI intervention. Children are neither contacted by the study team nor asked to participate directly in any study procedures.

\section{Practice and Ped/NP eligibility, recruitment and enrolment}

Practices and Ped/NPs were recruited through the AAP Pediatric Research in Office Settings (PROS) national primary care practice-based research network-among the longest-running paediatric primary care research networks in the USA ${ }^{23}$ - and the client database of PCC, an EHR vendor. A key inclusion criterion for practices was that they use PCC as their EHR vendor. The study team worked with PCC to modify their EHR to facilitate recruitment of eligible families, manage the delivery of the MI intervention (including tracking child behaviours), simplify billing and coding, and track child BMI values throughout the study. Practices were excluded from participating if they already offered a comprehensive weight loss programme or routine access to an RD for weight management. Ped/NPs were eligible to participate if they had been employed at a participating practice for at least 1 year and worked at least half-time. They were excluded if they had prior extensive experience with $\mathrm{MI}$ and/or had participated in the previous $\mathrm{BMI}^{2}$ trial or related pilot study. Each practice had 1-2 Peds/NPs who provided written informed consent to participate in the study (online supplementary material 1 ).

\section{Randomisation, concealment and blinding}

Randomisation occurred at the practice level by a statistician blinded to practice identity. Practices were stratified based on estimates of racial and ethnic composition and size of their patient population, and the nearest neighbours of that sort became a pair. Practices were then randomised within pairs to either intervention or usual care groups. Since the number of practices was small, it was necessary to restrict matching to the variables deemed to have the biggest potential impact on BMI. Other variables will be considered as covariates in multivariable models.

\section{Ped/NP and RD training Intervention}

Ped/NPs and RDs attended a 2-day interactive in-person training session led by the study principal investigator (senior author, who led the $\mathrm{BMI}^{2}$ trial and is experienced in MI). ${ }^{15} 162425$ The study team paid for all travel expenses (airfare, hotels and meals), but did not provide a stipend for participating in the training. Participants learnt and practised $\mathrm{MI}^{15} 16$ and reviewed general principles of behaviour therapy. ${ }^{26}$ Training also addressed billing and coding for paediatric weight management services and the study protocol. The MI training included a mix of didactic and experiential activities, following the reveal-practice-reveal model, with real-time constructive feedback. ${ }^{27}$ Core MI techniques taught included the use of effective questions, reflective listening, shared agenda setting, rolling with resistance, avoiding reactance, eliciting and reinforcing change talk, and structuring behaviour change plans. ${ }^{28}$ Since MI is applicable to many health behaviours, Ped/NPs were shown how it could be used for behaviours beyond nutrition and exercise. Continuing medical education (CME) and maintenance of certification (MOC) part II credits were available to participants after the training session. Paediatricians also had the option of completing an 18 month embedded quality improvement project for MOC part IV credits.

\section{Usual care}

Usual care Ped/NPs were instructed to continue their current weight management practices. They were trained in study procedures via phone, and given access to recorded webinars that reviewed Expert Committee 
Table 1 Schedule of enroment, training and intervention delivery in the $\mathrm{BMI}^{2+}$ study

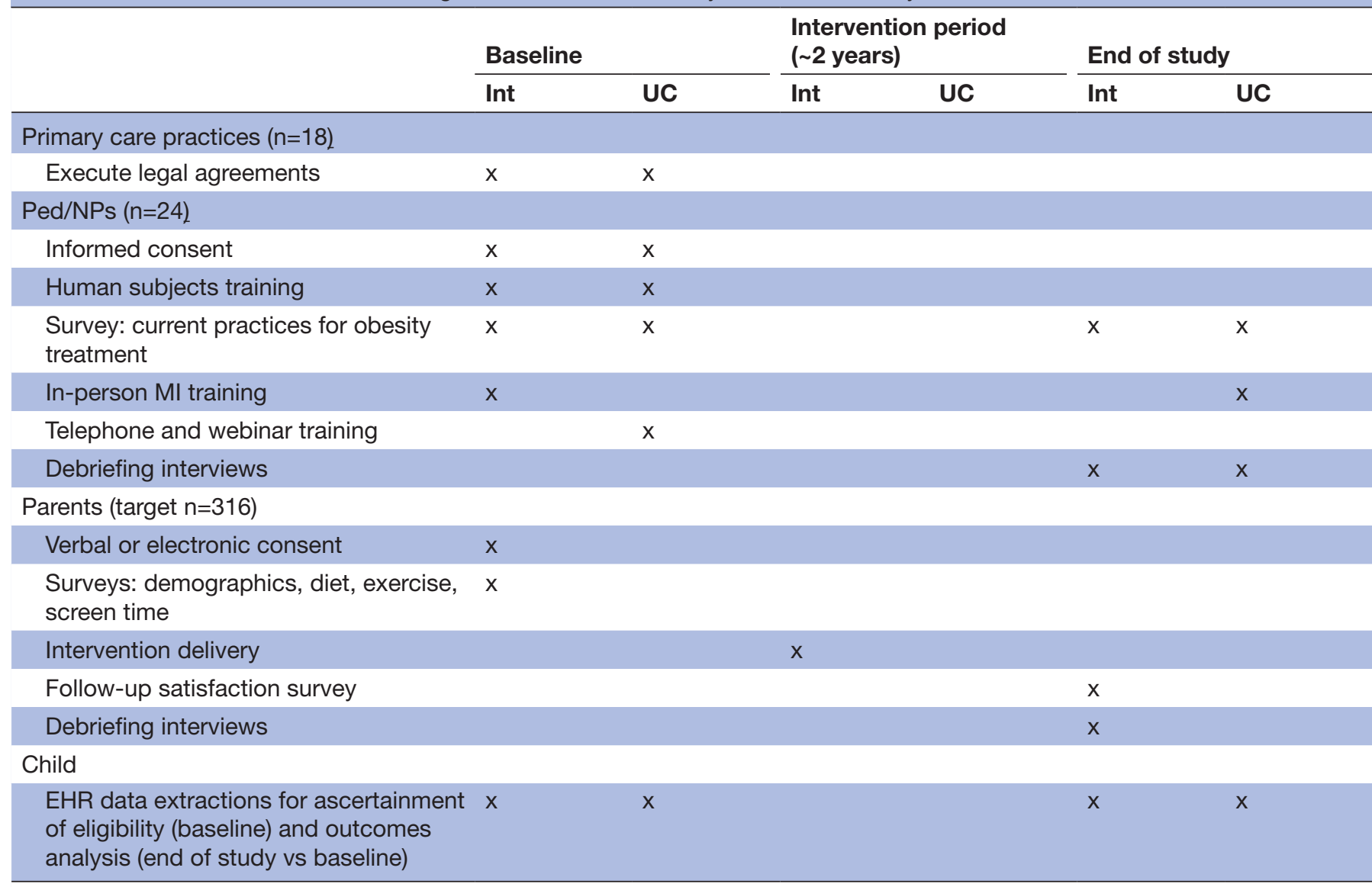

BMI, body mass index; EHR, electronic health recordlnt, intervention groupMI, motivational interviewing; ped/NP, paediatrician/nurse practitioner; UC, usual care group.

recommendations; the AAP obesity prevention, assessment and treatment algorithm for assessing and managing childhood obesity; and billing and coding tips. ${ }^{11} 122930 \mathrm{At}$ the end of the study, usual care Ped/NPs will be offered the opportunity to complete the full in-person MI and behavioural change training (travel costs, lodging and meals provided by the study) and receive $\mathrm{CME}$ and $\mathrm{MOC}$ II credits.

\section{Child eligibility}

In both the study groups, EHR data were used to identify eligible children at baseline and will be used to determine the study outcome, which is change in child BMI (per cent from the median BMI for age and sex) over the course of approximately 2 years.

\section{Intervention}

Parents of eligible children were identified using EHR data extracted from each intervention practice. Growth data (from the EHR) were cleaned using a previously validated method to identify implausible weight and height values based on deviations from expected growth trajectories. ${ }^{31}$ Eligible children:

- Were 3-11 years old on the date of the baseline data extraction (this age range differs from the prior efficacy trial in two ways: (1) we included 9-11 year old children because their eating behaviours are also largely determined by their parents and (2) we excluded 2-year-old children due to difficulty measuring height in this age group, as well as their more restricted diet and activity patterns).

- Had a BMI value for age and sex greater than or equal to the 85 th percentile (documented at the most recent office visit occurring during the 2 years prior to the baseline data extraction).

- Had their most recent health supervision visit during the 2 years prior to baseline with a participating Ped/ NP.

Children were excluded if they had any of the following documented in the EHR:

- Type I or type II diabetes.

- Daily or chronic use of medications known to affect growth and mood/behaviour (eg, growth hormones, SSRIs, stimulants).

- Use of atypical antipsychotics.

- Achronic, limiting, severe medical disorder, syndrome or other condition (eg, Down's syndrome, cerebral palsy), 


\section{Usual care}

The same procedures and criteria described above were used to identify eligible children in usual care practices.

\section{Parent recruitment and enrolment}

Recruitment and enrolment of parents of eligible children only occurred in intervention practices; these parents are receiving the intervention components described below.

\section{Intervention}

Intervention group Ped/NPs reviewed lists of their own eligible patients and made further exclusions based on their clinical judgement. Specifically, Ped/NPs were instructed to exclude families with social circumstances that would make recruitment inappropriate, such as homelessness or a recent death in the family, but not to exclude families simply because they thought they would decline to participate or otherwise not do well in the study. Families with multiple eligible children were asked to select one index child as the focus of their MI counselling sessions and survey responses. Parents were excluded from participating if they did not speak English or Spanish.

Contact information for parents of eligible children was transferred from PCC to the University of Michigan through a Business Associate Agreement (BAA— see the Ethics and Dissemination section). University of Michigan study team members mailed welcome letters to parents of eligible children. These letters contained an overview of the study, the web address of a study portal and a unique access code should the parent wish to access this portal to enrol online. Parent enrolment could occur in one of three ways: (1) parents could enrol in person at the child's doctor's office, (2) they could enrol over the phone during a call from a study RD working through the University of Michigan or (3) they could go to a study portal via the provided web address and unique access code to enrol online. Parents provided both informed consent and Health Insurance Portability and Accountability Act (HIPAA) authorisation-either verbally or electronically-at baseline (online supplementary material 2).

\section{Usual care}

There was no recruitment of, nor study team contact with parents in usual care practices.

\section{Intervention components}

Parents participating in the $\mathrm{BMI}^{2+}$ intervention are offered four key components: (1) in-person MI counselling by the Ped/NP; (2) remote, no cost, telephone MI counselling with a centrally employed, trained and supervised $\mathrm{RD}$; (3) text message reminders and behavioural reinforcement messages and (4) access to educational materials on a study portal.

\section{MI counselling}

Ped/NPs are asked to complete 4 MI-based in-person counselling sessions with enrolled parents over the course of approximately 2 years. These sessions can be scheduled as stand-alone office visits or can occur within the context of other scheduled appointments such as health supervision visits. It is up to the parent and Ped/NP to decide when to schedule these visits. RDs who are centrally employed, supervised, and located at the University of Michigan complete up to 6 MI-based telephone counselling sessions with enrolled parents during the same time period. The intervention is available in English and Spanish. Whereas the RD sessions are offered free of charge, Ped/NP sessions are billed using patient insurance, which may include co-pays and deductible payments.

MI counselling conversations between parents and Ped/ NPs and between parents and RD counsellors focus on one or more of the following target areas: snack foods, sweetened beverages, eating out, whole grains, fruits, vegetables, sweets and desserts, portion size, $\mathrm{TV} /$ screen time, video games and physical activity. Target areas are identified by parents when they complete a brief baseline survey-either online or over the phone with an RD-adapted from the prior $\mathrm{BMI}^{2}$ study. ${ }^{32}$ For each of the target areas, parents grade their child on a scale from A (great/healthy) to $\mathrm{F}$ (poor / unhealthy). Parent responses are then coded as red, yellow, or green. Ped/NPs have access to the letter grades and colour codes via their study portal (described in Intervention Components: Study Portals). Ped/NPs and RDs provide positive feedback for green behaviours, then collaboratively with the parent identify red or yellow behaviours that the parent would be willing to discuss and possibly modify. Ped/NPs are provided with summary notes from each RD counselling call, to assist them in their counselling and to enhance continuity of care. Parent education materials were developed for each target behaviour, and written in a style consistent with MI and self-determination theory. ${ }^{15} 33$ Ped/NPs and RDs provide these materials to enrolled parents at their discretion. Parents can also directly access these materials in their study portal (described in Intervention Components: Study Portals).

Given the real-world emphasis of this study, and distinct from the prior efficacy trial where we paid Ped/NPs for each MI counselling session, we trained Ped/NPs in billing and coding for weight management so they could bill naturally throughout the intervention. The study does not compensate Ped/NPs for providing MI counselling. However, practices in both groups receive payments for participating in the study: intervention practices receive US $\$ 2000-U S \$ 4000$, depending on how many of their eligible families enrol in the study, and usual care practices receive US\$1000.

\section{Text messages}

Enrolled parents receive tailored text messages between counselling calls aimed at enhancing motivation and reinforcing behavioural change. Tailored text messages are sent approximately weekly, to remind parents of their target areas and related goals, and some provide links to additional resources (see example message sequence in figure 3). In addition, parents receive automated text 


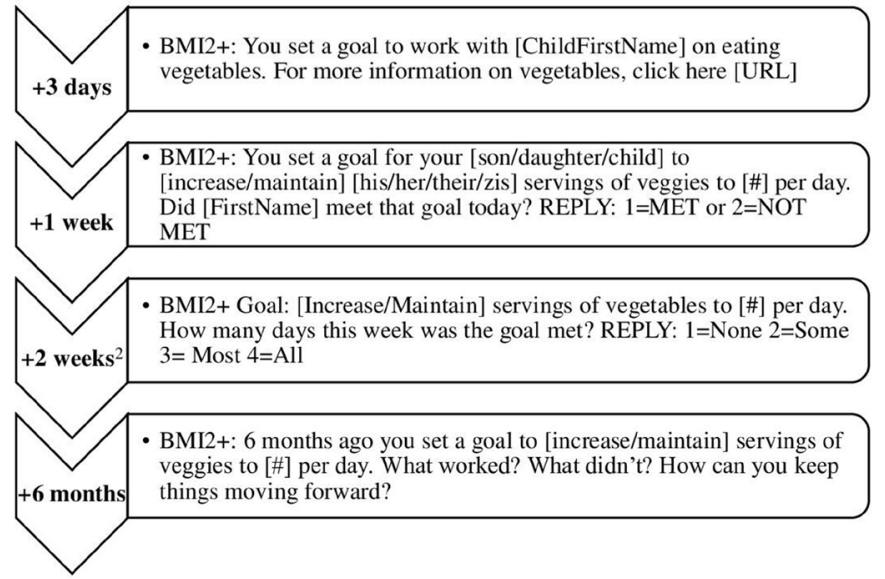

Figure 3 Text message sequence for an enrolled parent after selecting vegetable consumption as a goal area during a counselling call with an $R D^{1} .{ }^{1} \mathrm{RD}$ counselling call $=$ day 0 . ${ }^{2}$ repeated weekly for 1 month and monthly for 5 months. BMI, body mass index.

message reminders to complete surveys and schedule and complete counselling calls.

\section{Study portals}

Separate portals were developed for RDs, Ped/NPs and parents. The study portals are internet-based sites through which users can access intervention-related content that is securely stored on servers at the University of Michigan. RDs can use their portal to schedule, track, and document their consent and counselling calls with enrolled parents during the intervention period. Ped/NPs can use their portal to access study-related information, including enrolment history and clinical notes from RD counselling sessions, and supplemental MI training resources. Parents can use their portal to provide consent and enrol in the study, update their contact information, complete surveys, view summaries of past counselling calls with RDs, schedule future calls, and view and download educational materials. A chat feature within the portal allows for optional two-way messaging between parents and RDs, and between RDs and Ped/NPs.

\section{Intervention fidelity}

Standardised patient visits were conducted with all RDs and intervention group Ped/NPs at the end of in-person MI training. These encounters were audio-recorded and rated with a validated fidelity scale- the One-Pass coding system for evaluating healthcare professionals' competence in MI. ${ }^{34}$ Individual participants then received detailed confidential feedback from MI-trained study staff about their counselling encounter. Several other processes are in place to enhance MI skill acquisition and reduce skill atrophy:

- Intervention group Ped/NPs and RDs can access MI-related video clips and printed materials in their study portal (see above section: Intervention Components: Study Portals).

- The study team conducts optional group MI refresher calls with intervention group Ped/NPs by phone approximately every 3 months throughout the study, to review and practice core MI skills.

- Intervention group Ped/NPs are offered 1-3 individual standardised patient telephone sessions with feedback during the study.

- All RD counselling calls with enrolled parents are audio recorded, a random $10 \%$ sample are reviewed and rated with the One-Pass system by an MI supervisor, and feedback is provided to RDs as needed.

- RDs meet at least monthly with study staff at the University of Michigan to review and rehearse core MI skills.

\section{Study outcome}

The main outcome is change in child BMI, defined as per cent from the median BMI for age and sex over approximately 2 years. This metric recently has been shown to be a more reliable measure of adiposity than BMI z-score. ${ }^{35}{ }^{36}$ In addition, this metric does not have an upper limit and can be used to assess BMI in all children, even those with high BMI values. Furthermore, BMI z-score correlates poorly with adiposity measures such as circumferences, triceps skinfold and fat mass determined by dual-energy X-ray absorptiometry. ${ }^{37} \mathrm{New}$ guidelines for assessing adiposity change in children with elevated BMI values are expected in 2020 or 2021. Although we have currently selected \% from the median BMI for age and sex given recent publications showing its superiority, we will follow the final guidelines once they are issued.

At the conclusion of the trial, BMI values for eligible children will be extracted from the PCC EHR and cleaned for implausible values as described above (see the Methods and Analysis: Child Eligibility). ${ }^{31}$ We will characterise the outcome in two ways.

- First, we will select the child's BMI values that are closest to the 1-year and 2-year anniversary of the parent's consent/enrolment into the study (intervention) or their Ped/NPs baseline study procedures phone training (usual care). We will allow for a 3-month window around each time point. In this approach, up to $3 \mathrm{BMI}$ values will be analysed for each child.

- Second, we will use all available BMI values obtained from the EHR during the 2-year time frame for that child. We will model time as a continuous variable. In this approach, each child may have more than 3 BMI values available for analysis, and the timing of the assessment will be allowed to vary.

\section{Patient and public involvement}

At the end of the prior trial, 3 Ped/NPs, 7 RDs and 8 parents responded to questions about various aspects of their study experience. We applied those lessons learnt to inform the current study, including parents' desire for text message reminders and reinforcement messages, as well as greater availability of RDs for counselling sessions. ${ }^{20} 38$ Input from PROS member paediatricians was obtained 
during study design, before grant submission and during postaward protocol development.

\section{Data and statistical analysis}

Data for this trial are collected through surveys of Ped/ NPs and parents, and extraction of patient HIPAA-limited EHR and billing data.

\section{Intervention}

HIPAA-limited EHR and billing data were extracted at baseline and will be extracted again on completion of the intervention. At baseline, Ped/NPs completed a survey about their current practices for obesity treatment, which will be repeated at the end of the intervention. Enrolled parents completed baseline surveys (see Methods and Analysis: Intervention Components) to provide demographic information and identify target behaviours for Ped/NP and RD counselling. At the end of the intervention, parents will complete a follow-up survey about their engagement in the intervention, perception of impact of the intervention on their child's and family's lifestyle behaviours, as well as their satisfaction with Ped/ $\mathrm{NP}$ and RD counselling. Parents will receive an incentive after completing the follow-up survey-this is the only compensation that they receive during the course of the study. Ped/NPs will be contacted at the end of the study to complete a semistructured interview to elucidate key issues and opportunities for future enhancements.

\section{Usual care}

The data collection procedures and sources described above are identical for Usual Care practices, except that there is no parent contact of any kind in Usual Care practices (no parent surveys nor compensation).

Mixed effects linear regression will be used to model the effect of the intervention on change in child per cent from median BMI for age and sex over approximately 2 years. To control for cluster randomisation effects, SAS/ PROC MIXED will be used with practice treated as a random effect. Potential covariates and effect modifiers include child sex, age, and ethnicity/race, time to completion of RD calls, and parent use of the study website and other features therein (eg, scheduling function, diaries and handouts, two-way chat feature with RD).

For our primary outcome, we will compare change in per cent from median BMI for age and sex among children of enrollees in the study (intervention) compared with eligible children in usual care practices (intentionto-treat analysis). The secondary aims are to compare changes in \% from median BMI for age and sex in (1) the subgroup of enrolled patients of participating Ped/NPs who actively participated in the intervention (defined as receipt of at least $50 \%$ of the total MI counselling sessions from Ped/NPs and RDs) versus all eligible children in usual care practices (analysis of completers per protocol) and (2) all eligible children of participating Ped/NPs in intervention practices, even if the child's parent did not enrol in the study, versus all eligible children in usual care practices (population-level analysis). In addition to defining active parent engagement in the intervention as receipt of at least $50 \%$ of MI counselling sessions, we will also examine whether there is a linear doseresponse relationship between the number of MI counselling sessions received and study outcomes. We will also consider using multiple imputation of missing BMI score values depending on the missingness pattern in the final data set. ${ }^{39}$

\section{Sample size}

The study was initially powered to detect an effect of 0.10 BMI z-score units (the original metric used in the grant application) between intervention and usual care groups at 2-year follow-up, with an assumed SD of 0.40 , power of 0.80 , and two-tailed alpha of 0.05 . This equates to a standardised effect of $0.25(0.10 / 0.40)$. To convert this same effect size to our revised outcome, per cent from median BMI for age and sex, we used data from Freedman et al. ${ }^{35}$ Assuming our sample (all of whom are above the 85th percentile) will average around $30 \%-40 \%$ above the median (which equates to the 97 th percentile), with an SD of 20-25, this equates to a change of approximately 5-6 percentage-from-median units. We will log transform our primary outcome variable as needed.

Sample size estimates account for practice-level clustering, ${ }^{40}$ assuming a practice-level intraclass correlation coefficient between 0.001 and 0.03 . Based on these assumptions, we required seven practices per study group (14 total, although 2 additional practices were recruited in each study group to account for possible practice attrition) and an average of about 35 enrolled parents per intervention practice (target $\mathrm{n}=316$ ).

\section{ETHICS AND DISSEMINATION}

A series of legal agreements allow for the transfer of data and protected health information between multiple collaborating institutions during the study:

- A data use agreement (DUA) allows for the transfer of HIPAA limited datasets from the EHR vendor to the study team.

- A data transfer agreement is used in conjunction with parent HIPAA authorisation to allow for the free exchange of study-related and clinical information between intervention practices and RDs.

- A BAA allows for the transfer of patient contact information, without HIPAA authorisation, from the EHR vendor to the study team for the purposes of study recruitment.

Modifications to the protocol are tracked centrally by the study team via institutional review board (IRB) amendment approval dates and dates of modification to ClinicalTrials.gov.

Study team members at the University of Michigan, the CHOP, and the AAP will have access to the final trial dataset. 
IRB approval was obtained from the AAP, the IRB of record for the trial. The University of Michigan formally relied on the AAP IRB via an IRB Authorisation Agreement and Streamlined, Multisite, Accelerated Resources for Trials (SMART) IRB roles and responsibilities grid. CHOP handles data deemed 'not readily' identifiable by the CHOP IRB, and those activities are therefore exempt. All Ped/NPs provided written informed consent, and intervention group parents provided consent and HIPAA authorisation verbally or electronically prior to enrolment.

Practices, Ped/NPs and parents can withdraw from the study at any time, as explicitly stated in all consents. If a parent withdraws, counselling calls end, they no longer receive surveys or study text/email messages, and they no longer have access to their study portal. The parent and Ped/NP decide together whether to continue with office-based MI counselling. All data that the parent has provided up to the date of withdrawal continues to be stored and analysed, and HIPAA limited data (including their child's BMI) continues to be extracted through the end of the study as a whole, as permitted in the DUAs.

RDs systematically assess the potential for disordered eating behaviours and/or excessive weight loss during their counselling calls with enrolled parents. Study progress (recruitment, retention) and potential adverse events are monitored and reviewed at least annually by a data safety and monitoring board throughout the trial.

Trial results will be presented to all participating practices and Ped/NPs, and disseminated through peerreviewed publications, conference presentations and pertinent AAP channels. The AAP develops evidencebased clinical practice guidelines for a wide range of child health topics, and assists paediatricians as they implement these recommendations at the point of care. ${ }^{41}{ }^{42}$ If the $\mathrm{BMI}^{2+}$ intervention is effective, results could inform future AAP policy, resources, and tools regarding paediatric weight management.

\section{DISCUSSION}

The current $\mathrm{BMI}^{2+}$ effectiveness trial builds on the prior evidence of statistically significant and clinically meaningful reductions in BMI percentile among children whose parents received MI counselling from Ped/NPs and RDs, compared with usual care, over approximately 2 years. ${ }^{41}$ The current study includes several enhancements, including (1) moving the RD counselling to a centralised telephonic system, (2) adding text messaging to enhance parent engagement and reduce attrition, (3) use of study portals to schedule, track and document consent and counselling calls (RDs), and provide secure interfaces for accessing clinical and research content (RDs, Ped/NPs, and parents), (4) using EHRs for eligibility determination, documentation, intervention delivery and outcomes analyses and (5) analysis of intervention effectiveness at the population level, in addition to the evaluation among enrollees.
To date, the study team has successfully enrolled paediatric primary care practices, provided training to PCPs/ NPs in the intervention and usual care groups, extracted and cleaned baseline EHR data, completed parent recruitment and enrollment, and begun delivery of the intervention. Results are anticipated in late 2021 or early 2022, after the intervention is complete and data are collected and analysed.

There are several limitations. First, the study relies on $\mathrm{BMI}$ as the only measure of intervention effectiveness. We considered using other clinical biomarkers (eg, Hemoglobin A1c (HbA1c) or blood pressure) in addition to BMI, but they are frequently missing in EHR data, and the study or the families themselves may have had to cover their cost. We also considered using self-reported dietary intake measures or accelerometers to measure physical activity. However, given the budget, these were not financially feasible. We also had concerns that adding assessments would impose a burden that might discourage enrolment into the trial and subsequent retention. Second, we excluded children with some co-occurring disease, such as diabetes, which limits generalisability. Third, while Ped/NPs in intervention practices reviewed lists of eligible children and made further exclusions based on social circumstances, Ped/NPs in usual care practices did not do so (since there was no contact with children or families in those practices). Finally, the trial was restricted to practices that used one specific EHR vendor, which may limit generalisability.

\section{Author affiliations}

${ }^{1}$ Primary Care Research, American Academy of Pediatrics, Itasca, Illinois, USA ${ }^{2}$ Pediatric Research in Office Settings, American Academy of Pediatrics, Itasca, Illinois, USA

${ }^{3}$ School of Public Health, University of Michigan, Ann Arbor, Michigan, USA

${ }^{4}$ Physician's Computer Company, Winooski, Vermont, USA

${ }^{5}$ Department of Paediatrics, University of California Los Angeles, Los Angeles, California, USA

${ }^{6}$ Department of Hospital Biostatistics, The Rockefeller University, New york City, New york, USA

${ }^{7}$ Department of Paediatrics, Children's Hospital of Philadelphia, Philadelphia, Pennsylvania, USA

${ }^{8}$ Departments of Pediatrics and Population and Family Health, Columbia University Irving Medical Center, New york City, New york, USA

Acknowledgements The authors thank the PROS network primary care paediatricians and nurse practitioners for their participation in the $\mathrm{BMI}^{2+}$ study. The authors also thank all participating parents.

Contributors KR is the principal investigator; as such, he conceived, designed and oversaw the study. Trial conduct and recruitment was carried out by MEW, ED, KRS SC, JS, DH, LPS, AGF, MSS and HW. TP conducted all data extractions at PCC. RV and RWG provided expertise in statistics and data analytics. All authors reviewed and approved the final manuscript.

Funding This work was supported by the National Heart Lung and Blood Institute (NHLBI) grant number 1R01HL128231-01A1. Additional infrastructure support was provided by the American Academy of Pediatrics and the Health Resources and Services Administration (HRSA) of the US Department of Health and Human Services (HHS) under UA6MC15585 - National Research Network to Improve Children's Health.

Disclaimer The information, content and/or conclusions are those of the author(s) and should not be construed as the official position or policy of, nor should any endorsements be inferred by HRSA, HHS or the US Government. 
Competing interests None declared

Patient consent for publication Not required.

Provenance and peer review Not commissioned; externally peer reviewed.

Open access This is an open access article distributed in accordance with the Creative Commons Attribution Non Commercial (CC BY-NC 4.0) license, which permits others to distribute, remix, adapt, build upon this work non-commercially, and license their derivative works on different terms, provided the original work is properly cited, appropriate credit is given, any changes made indicated, and the use is non-commercial. See: http://creativecommons.org/licenses/by-nc/4.0/.

ORCID iD

Margaret E Wright http://orcid.org/0000-0002-4410-4157

\section{REFERENCES}

1 Ogden CL, Fryar CD, Hales CM, et al. Differences in obesity prevalence by demographics and urbanization in US children and adolescents, 2013-2016. JAMA 2018;319:2410-8.

2 Skinner AC, Ravanbakht SN, Skelton JA, et al. Prevalence of obesity and severe obesity in US children, 1999-2016. Pediatrics 2018;141:e20173459.

3 Weaver RG, Brazendale K, Hunt E, et al. Disparities in childhood overweight and obesity by income in the United States: an epidemiological examination using three nationally representative datasets. Int J Obes 2019;43:1210-22.

4 Singh AS, Mulder C, Twisk JWR, et al. Tracking of childhood overweight into adulthood: a systematic review of the literature. Obes Rev 2008;9:474-88.

5 Kelsey MM, Zaepfel A, Bjornstad P, et al. Age-Related consequences of childhood obesity. Gerontology 2014;60:222-8.

6 Ul-Haq Z, Mackay DF, Fenwick E, et al. Meta-Analysis of the association between body mass index and health-related quality of life among children and adolescents, assessed using the pediatric quality of life inventory index. J Pediatr 2013;162:280-6.

7 Buttitta M, Iliescu C, Rousseau A, et al. Quality of life in overweight and obese children and adolescents: a literature review. Qual Life Res 2014;23:1117-39.

8 Obesity trends in the United States. J Nat/ Cancer Inst 2016;108.

9 Bright Futures/American Academy of Pediatrics. Bright Futures. In: Hagan JF, Shaw JS, Duncan PM, eds. Guidelines for health supervision of infants, children, and adolescents. 4 ed. Elk Grove Village, IL: Bright Futures/American Academy of Pediatrics, 2017.

10 Freed GL, Dunham KM, Gebremariam A, et al. Research advisory committee of the American Board of Pediatrics. Which pediatricians are providing care to America's children? An update on the trends and changes during the past 26 years. J Pediatr 2010;157:148-52.

11 Barlow SE, Expert Committee. Expert Committee recommendations regarding the prevention, assessment, and treatment of child and adolescent overweight and obesity: summary report. Pediatrics 2007;120 Suppl 4:S164-92.

12 Spear BA, Barlow SE, Ervin C, et al. Recommendations for treatment of child and adolescent overweight and obesity. Pediatrics 2007;120 Suppl 4:S254-88.

13 O'Connor EA, Evans CV, Burda BU, et al. Screening for obesity and intervention for weight management in children and adolescents: evidence report and systematic review for the US preventive services Task force. JAMA 2017;317:2427-44.

14 Miller WR, Rollnick S. Motivational interviewing: preparing people to change addictive behavior. New York, London: Guilford, 1991.

15 Resnicow K, McMaster F. Motivational interviewing: moving from why to how with autonomy support. Int J Behav Nutr Phys Act 2012;9:19.

16 Resnicow K, Davis R, Rollnick S. Motivational interviewing for pediatric obesity: conceptual issues and evidence review. J Am Diet Assoc 2006;106:2024-33.

17 Kraft C, Frintner MP, Goodman AB, et al. Pediatrician-perceived preparedness in motivational interviewing (MI) and perceived effectiveness in counseling children with obesity presented at the 2018 pediatric academic societies annual meeting in Toronto, Canada. Available: https://ihcw.aap.org/Documents/Results/PedP erceivedPreparednessMI.pdf

18 Belay B, Frintner MP, Liebhart JL, et al. Us pediatrician practices and attitudes concerning childhood obesity: 2006 and 2017. J Pediatr 2019;211:78-84.
19 National Heart, Lung, and Blood Institute. BMI2: Brief Motivational Interviewing to Reduce Child BMI - to Dr. Ken Resnicow, 2011.

20 Resnicow K, McMaster F, Bocian A, et al. Motivational interviewing and dietary counseling for obesity in primary care: an RCT. Pediatrics 2015;135:649-57.

21 Reinehr T, Kiess W, Kapellen T, et al. Insulin sensitivity among obese children and adolescents, according to degree of weight loss. Pediatrics 2004;114:1569-73.

22 Ford AL, Hunt LP, Cooper A, et al. What reduction in BMI SDS is required in obese adolescents to improve body composition and cardiometabolic health? Arch Dis Child 2010;95:256-61.

23 Fiks AG, Scheindlin B, Shone L. 30Th anniversary of pediatric research in office settings (pros): an invitation to become engaged. Pediatrics 2016;138:peds.2016-1126.

24 Resnicow K, Sonneville KR, Naar S. The heterogeneity of $\mathrm{Ml}$ interventions studies for treatment of obesity. Pediatrics 2018;142:peds.2018-2471.

25 Resnicow K, Dilorio C, Soet JE, et al. Motivational interviewing in health promotion: it sounds like something is changing. Health Psychol 2002;21:444-51.

26 Naar-King S, Safren SA. Motivational interviewing and CBT: combining strategies for maximum effectiveness. NewYork, NY: The Guilford Press, 2017.

27 Miller WR, Rollnick S. Motivational interviewing: helping people change. 3rd ed. New York: Guilford Press, 2013.

28 Resnicow K, Gobat N, Naar S. Intensifying and igniting change talk in motivational interviewing: a theoretical and practical framework. Eur $J$ Health Psychol 2015;17:102-10.

29 Institute for Healthy Childhood Weight, American Academy of Pediatrics. Algorithm for the assessment and management of childhood obesity in patients 2 years and older. Available: https:// ihcw.aap.org/Documents/Assessment and Management of Childhood Obesity Algorithm_FINAL.pdf

30 US Preventive Services Task Force, Grossman DC, Bibbins-Domingo $\mathrm{K}$, et al. Screening for obesity in children and adolescents: US preventive services Task force recommendation statement. JAMA 2017;317:2417-26.

31 Daymont C, Ross ME, Russell Localio A, et al. Automated identification of implausible values in growth data from pediatric electronic health records. J Am Med Inform Assoc 2017;24:1080-7.

32 Resnicow K, McMaster F, Woolford S, et al. Study design and baseline description of the BMI2 trial: reducing paediatric obesity in primary care practices. Pediatr Obes 2012;7:3-15.

33 Vansteenkiste M, Williams GC, Resnicow K. Toward systematic integration between self-determination theory and motivational interviewing as examples of top-down and bottom-up intervention development: autonomy or volition as a fundamental theoretical principle. Int J Behav Nutr Phys Act 2012;9:23.

34 McMaster F, Resnicow K. Validation of the one pass measure for motivational interviewing competence. Patient Educ Couns 2015;98:499-505.

35 Freedman DS, Woo JG, Ogden CL, et al. Distance and percentage distance from median BMI as alternatives to BMI z score. Br J Nutr 2019:1-8.

36 Dietz WH. Time to adopt new measures of severe obesity in children and adolescents. Pediatrics 2017;140:peds.2017-2148.

37 Freedman DS, Butte NF, Taveras EM, et al. Bmi z-Scores are a poor indicator of adiposity among 2- to 19-year-olds with very high BMls, NHANES 1999-2000 to 2013-2014. Obesity 2017;25:739-46.

38 Resnicow K, Harris D, Wasserman R, et al. Advances in motivational interviewing for pediatric obesity: results of the brief motivational interviewing to reduce body mass index trial and future directions. Pediatr Clin North Am 2016;63:539-62.

39 Rubin DB. Multiple imputation for nonresponse in surveys. Hoboken, N.J: Wiley-Interscience, 2004.

40 Murray DM. Design and analysis of community trials: lessons from the Minnesota heart health program. Am J Epidemiol 1995;142:569-75.

41 American Academy of Pediatrics Steering Committee on Quality Improvement and Management, Shiffman RN, Marcuse EK, et al. Toward transparent clinical policies. Pediatrics 2008;121:643-6.

42 American Academy of Pediatrics Steering Committee on Quality Improvement and Management. Classifying recommendations for clinical practice guidelines. Pediatrics 2004;114:874-7. 